\title{
Collection of Sixteen High-Quality Human Head CAD Models Generated with SimNIBS 2.1 Using Connectome Subject Data within MATLAB ${ }^{\circledR}$ Platform
}

\author{
Aung Thu Htet ${ }^{1}$, Gregory M. Noetscher ${ }^{1,2}$, Edward H. Burnham ${ }^{1}$, Aapo Nummenmaa ${ }^{3}$
} and Sergey N. Makarov ${ }^{1-3}$

${ }^{1}$ ECE Department, Worcester Polytechnic Inst., Worcester, MA 01609 USA

${ }^{2}$ NEVA Electromagnetics, LLC, Yarmouth Port, MA 02675 USA

${ }^{3}$ Athinoula A. Martinos Center for Biomedical Imaging, Massachusetts General Hospital, Charlestown, MA 02129 USA

\begin{abstract}
The goal of this study is to introduce a collection of sixteen high-resolution, 2-manifold CAD compatible head models within the MATLAB platform available to all interested parties for electromagnetic and acoustic simulations. Each model contains skin, skull, CSF, GM, cerebellum, WM, and ventricles head compartments and possesses an "onion" topology: the grey matter shell is a container for white matter, ventricles, and cerebellum objects, the CSF shell contains the grey matter shell, the skull shell contains the CSF shell, and finally the skin or scalp shell contains the skull shell. The models are fully compatible with ANSYS ED FEM software, CST Studio Suite, Sim4Life/SEMCAD software, and other electromagnetic software packages.

The collection is based on MRI data from the Human Connectome Project (HCP) segmented using the SimNIBS 2.1/2.1.1 processing pipeline. The average number of triangular surface facets in a model is 866,000 , the average triangle quality is 0.25 , the average edge length is 1.48 $\mathrm{mm}$, and the average surface mesh density or resolution is 0.57 points per $\mathrm{mm}^{2}$. If necessary, a finer model mesh can be created for every head using available MATLAB tools.
\end{abstract}

Keywords: Computational Models, Neurostimulation, Electromagnetic Modeling 


\section{Introduction}

For all three chief neurostimulation modalities - transcranial magnetic stimulation (TMS), transcranial electric stimulation (TES), and intracortical microstimulation (ICMS) - numerical computation of the electric fields within a patient-specific head model is the major and often only way to foster spatial targeting and obtain a quantitative measure of the required stimulation dose [1]. An important component of any simulation setup is the human head model itself, either a voxel model or a computer aided design (CAD) model represented by a set of solid objects [2].

Several open-source software packages currently exist that allow for automatic and semiautomatic generation of anatomically realistic CAD head models from individual MRI datasets. A comprehensive review can be found in [3]. Of specific note is the SimNIBS package [4]-[8] that uses FreeSurfer software [9],[10] for brain compartment segmentation (grey matter, white matter, cerebellum) and FSL [11],[12] for compatible skull/cerebrospinal fluid/ventricles segmentation when a combination of T1- and T2-weighted images is used as input.

At the same time, generic computational cranium models and collections of those continue to remain important for population-based studies as well as for testing accuracy, speed, and performance of various numerical algorithms and software packages. The most detailed anatomical CAD head model to date is arguably the MIDA model [13].

The Human Connectome Project (HCP) [14] provides a unique, open source, large-scale collection of about 1200 human head T1/T2 image datasets obtained with the isotropic resolution of $0.7 \mathrm{~mm}$, along with the corresponding diffusion tensor imaging (DTL) data. Fifty such datasets have recently been segmented by E.G. Lee and colleagues [15],[16],[17] using SimNIBS, the well-known, open-source transcranial brain stimulation modeling software [4]-[8]. The resulting fifty CAD models, known as the Population Head Model Repository, are available from the website of the IT'IS Foundation, Switzerland [18].

The goal of this study is to provide a collection of sixteen high-resolution, 2-manifold, CAD ready head models within the MATLAB $^{\circledR}$ platform that are also fully compatible with ANSYS ED FEM software, CST Studio Suite, Sim4Life/SEMCAD software, and other electromagnetic software packages. Similar to the Population Head Model Repository [15],[16],[17] we also use the SimNIBS pipeline for automatic segmentation of structural T1 and T2 weighted MRI images of the Connectome database [14]. We extract the following major compartments: skin (or scalp), skull (outer surface), cerebrospinal fluid or CSF, which is simultaneously the inner surface of the skull, grey matter, cerebellum, white matter, and the ventricles. The entire model possesses an "onion" topology: the grey matter shell is a container for white matter, ventricles, and cerebellum objects, the CSF shell contains the grey matter shell, the skull shell contains the CSF shell, and finally the skin or scalp shell contains the skull shell.

In contrast to the Population Head Model Repository [15],[16], we did not perform any mesh postprocessing. Instead, we output the original data obtained with the most recent versions SimNIBS v. 2.1 and SimNIBS v. 2.1.1. In the Population Head Model Repository, this postprocessing performed with MeshMixer was focused on the skin (reducing mesh resolution) and the skull, where some models appeared to have very isolated divots that decreased the skull's thickness, replacing it with more skin [17]. This postprocessing resulted in the appearance of non-manifold meshes which may be undesirable. Therefore, we independently segmented all fifty models of the Population Head Model Repository. After that, we have chosen sixteen models with a smooth skull surface. We also kept the high-resolution skin surface desirable for transcranial electric stimulation (TES) modeling and Electroencephalogram (EEG) modeling. 


\section{Materials and Methods}

\subsection{MRI Data}

The MRI data were kindly provided by the HCP [14], WU-Minn Consortium (Principal Investigators: David Van Essen and Kamil Ugurbil; 1U54MH091657) funded by the 16 NIH Institutes and Centers that support the NIH Blueprint for Neuroscience Research; and by the McDonnell Center for Systems Neuroscience at Washington University. Users can sign up for a ConnectomeDB account and, after signing the Open Access Data Use Terms, can download more HCP data themselves as described on pp. 24-25 of the S1200 Reference Manual [21].

\subsection{Segmentation}

The T1 and T2 image files of the Connectome database [14] were used as input for the SimNIBS segmentation pipeline. For subject 101309, as an example, the command used was: mri2mesh --all 101309 T1w.nii.gz T2w.nii.gz where 101309 in this command is the unique Connectome's subject ID. We updated from SimNIBS 2.1 to SimNIBS 2.1.1 at some time in the middle of the segmentation process. Subjects with IDs equal to or lower than 111716 were generated using SimNIBS 2.1 whereas subjects with IDs higher than that were segmented using SimNIBS 2.1.1.

Only the option --all was used. The option is "to run all reconstruction steps including volume meshing" according to mri2mesh documentation [19]. The segmentation time varies with each subject. Generally, segmentation of each head took from 10 to 12 hours using an Intel Xeon E5-2690 0 CPU 2.90GHz Linux server with 192 Gbytes of RAM.

The mri2mesh tool automatically generated the STL files for every individual head compartment. These files have been processed in MATLAB ${ }^{\circledR}$ and in a commercial FEM electromagnetic software package ANSYS Electronics Desktop 2018.1.

\subsection{Subject selection}

After independently segmenting all fifty subjects used in the Population Head Repository [15][18], a visual inspection of the skull segmentation quality has been performed and the "acceptable" models have been retained. Figure 1 shows examples of acceptable and unacceptable outer skull shells.
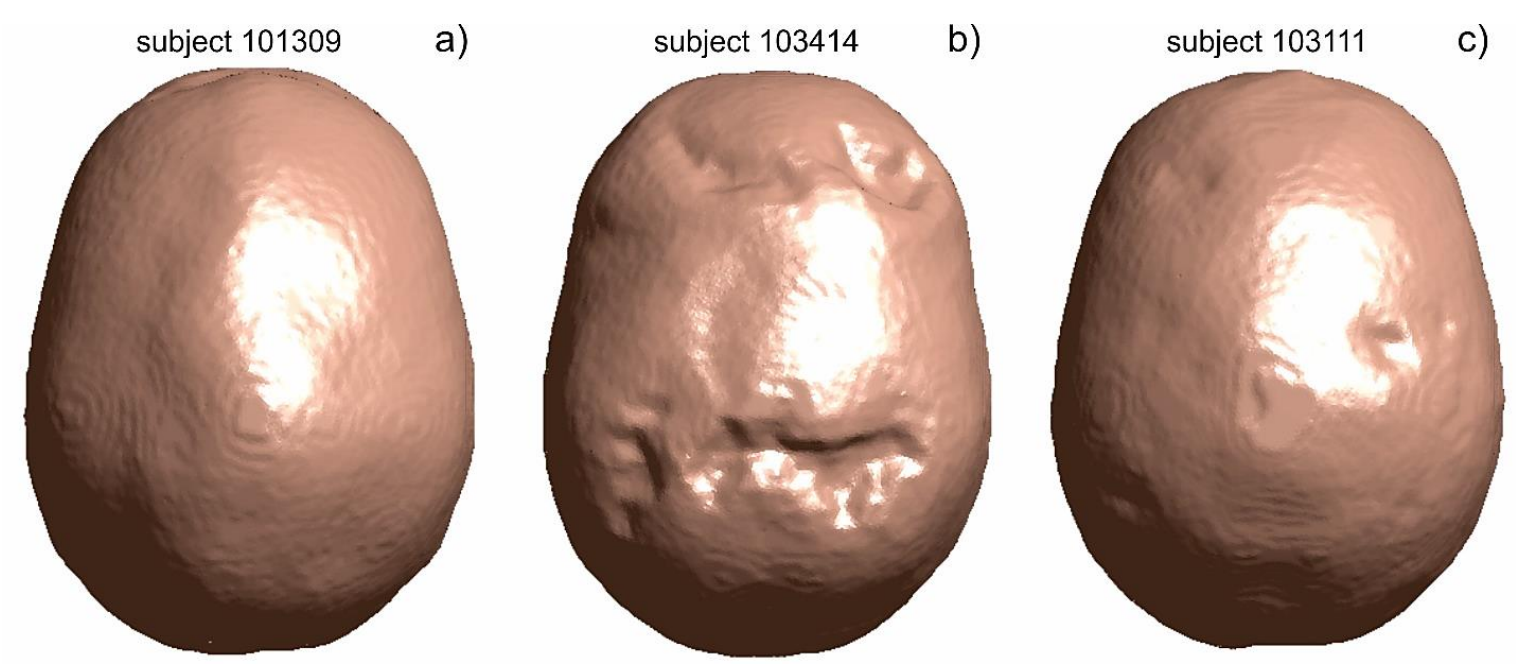

Figure 1. a) - Acceptable outer skin shell; b) - unacceptable outer skin shell; c) - unacceptable outer skin shell. 
As a result, we ended up with sixteen acceptable models from the pool of fifty segmented models in total. We emphasize that an inspection of the CSF (or inner skull) surface and other compartments has also been performed.

\subsection{Subject data}

If restricted subject data (e.g. exact ages of subjects, health or substance use data, self-report psychiatric symptom data) is required, HCP needs to be informed and the Washington University IRB needs to be involved in the decision to allow or deny this.

\section{Results}

\subsection{Data organization}

Figure 2 shows the data organization chart. Every folder has the data for a specific head model: the folder name is the Connectome's head ID. The data are given in common STL (STereoLithography) format and in MATLAB format (array of nodes $P$, array of facets $t$, and array of outer normal vectors normals).
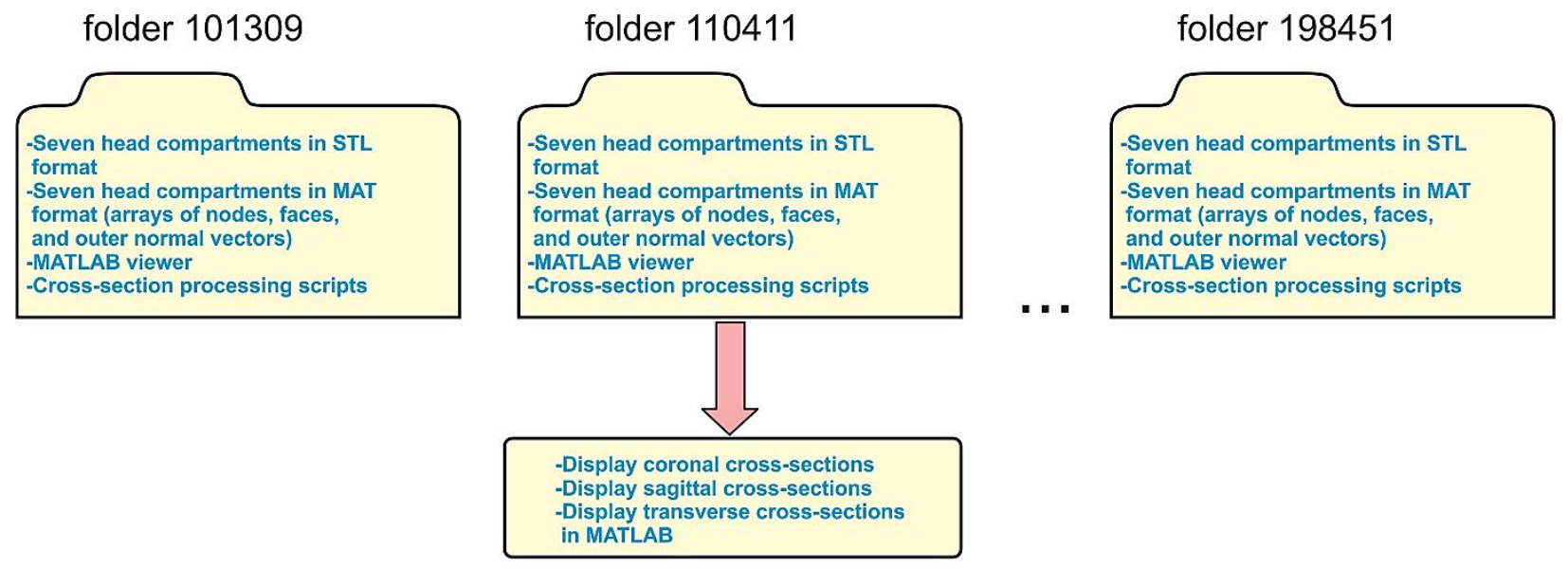

Figure 2. Data organization chart.

Every folder also has a number of simple MATLAB processing scripts which allow for a 3D visualization and model cross-section identification in any principal plane. Furthermore, the mesh characteristics can be computed as described below. As an example, Figure 3 shows three cross-section cuts for subject \# 110411.

An outer folder has the STL to MAT conversion tools created by Dr. Pau Micó, Universitat Politècnica de València, Spain, and available from MATLAB Central [22]. Additionally, we also present a wrapper that collects and processes data from all sixteen head models.

\subsection{Topological mesh characteristics}

Table 1 reports the number of triangular faces in every head compartment of every dataset. The averaged total number of facets per head dataset is 866,000. Table 2 reports the mesh quality factor, $Q$ [20], for every head compartment of every dataset. The average mesh quality over all compartments of all datasets is remarkably high; it is equal to 0.25 . The skin and CSF (inner skull) shells possess the lowest mesh quality of the assembly. Table 3 reports the average edge length, $l, \mathrm{~mm}$ of the triangular surface mesh for every head compartment of every dataset. The average edge length over all compartments of all datasets is $1.5 \mathrm{~mm}$. Finally, Table 4 reports an average mesh resolution, $R$, in points $/ \mathrm{mm}^{2}$ for every head compartment of every dataset. This 
parameter is in particular used by the developers of SimNIBS software. The average mesh resolution over all compartments of all datasets is 0.6 points per $\mathrm{mm}^{2}$.

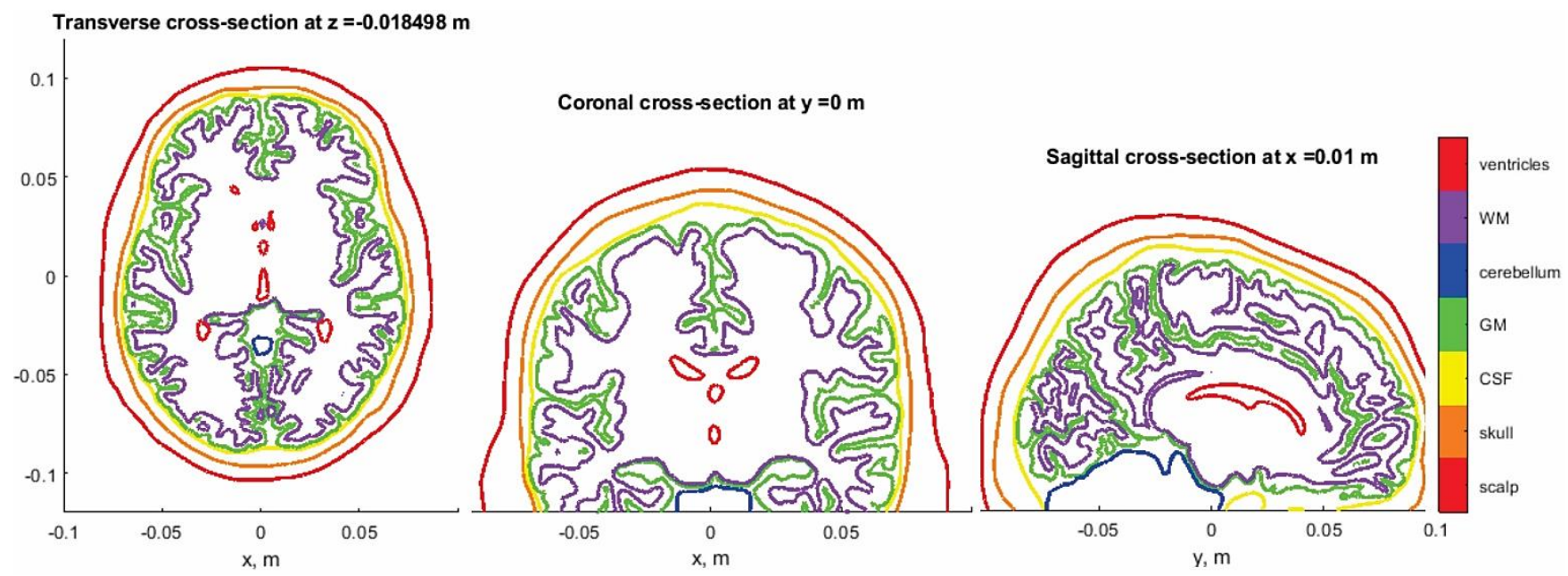

Figure 3. Three cross-section cuts for subject \# 110411.

\subsection{Specific conditions for CAD models}

The following two conditions are required for a true CAD human body model [20]: (1) a 3D triangular mesh representing a solid object must not have holes; (2) the surface of a well-behaved triangular mesh in 3D must satisfy the so-called manifold condition. A mesh is 2-manifold if every node of the mesh has a disk-shaped neighborhood of triangles. This neighborhood can be continuously deformed to an open disk. Every edge of a 2-manifold mesh is a manifold edge with only two attached triangles. All other meshes are non-manifold meshes and are not suitable for FEM analysis. Figure 4 gives examples of a manifold edge, a non-manifold edge and a nonmanifold mesh with a non-manifold node.

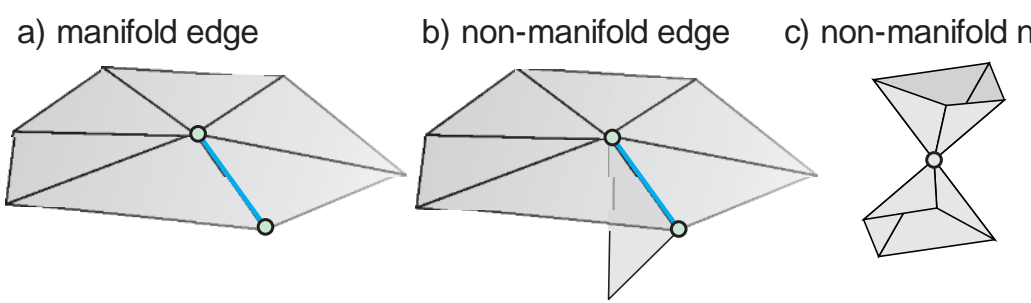

Figure 4. a) - Examples of a manifold edge; b) - non-manifold edge, and c) - non-manifold node.

In order to check the CAD conditions, we applied the mesh checker of the commercial FEM software ANSYS Electronics Desktop (ANSYS Maxwell) 2018.1 with the check option "strict". Each head compartment of every dataset has been checked by creating a separate ANSYS project and importing the corresponding STL data. The results showed that all the surface meshes passed the ANSYS check without errors.

\subsection{Comparison with the Population Head Repository}

As compared to the Population Head Repository [18], we have nearly doubled the resolution and significantly increased triangle quality for the skin surface. Also, all present CAD meshes are 2manifold, which is not the case for the Population Head Repository. 
Table 1. Number of triangular faces in each head compartment of every dataset.

\begin{tabular}{|c|c|c|c|c|c|c|c|c|c|}
\hline$\#$ & Head ID & $\begin{array}{c}\text { Faces, } \\
\text { skin }\end{array}$ & $\begin{array}{c}\text { Faces, } \\
\text { skull }\end{array}$ & $\begin{array}{c}\text { Faces, } \\
\text { CSF }\end{array}$ & $\begin{array}{c}\text { Faces, } \\
\text { GM }\end{array}$ & $\begin{array}{c}\text { Faces, } \\
\text { cerebel. }\end{array}$ & $\begin{array}{c}\text { Faces, } \\
\text { WM }\end{array}$ & $\begin{array}{c}\text { Faces, } \\
\text { ventricles }\end{array}$ & $\begin{array}{c}\text { Faces, } \\
\text { total }\end{array}$ \\
\hline 1 & 101309 & 119990 & 119996 & 119994 & 222106 & 17136 & 235732 & 14958 & 849912 \\
\hline 2 & 110411 & 119990 & 119992 & 119994 & 245886 & 17140 & 235608 & 14968 & 873578 \\
\hline 3 & 117122 & 119992 & 119992 & 119994 & 240888 & 17152 & 235350 & 15004 & 868372 \\
\hline 4 & 120111 & 119986 & 119992 & 119994 & 219610 & 17150 & 235120 & 12400 & 844252 \\
\hline 5 & 122317 & 119986 & 119978 & 119990 & 245564 & 17142 & 235530 & 15004 & 873194 \\
\hline 6 & 122620 & 119986 & 119988 & 119990 & 237744 & 17158 & 235420 & 15002 & 865288 \\
\hline 7 & 124422 & 119990 & 119988 & 119992 & 251218 & 17146 & 235348 & 14998 & 878680 \\
\hline 8 & 128632 & 119974 & 119990 & 119994 & 232810 & 17140 & 235346 & 14964 & 860218 \\
\hline 9 & 130013 & 119990 & 119988 & 119996 & 238814 & 17142 & 235934 & 14994 & 866858 \\
\hline 10 & 131722 & 119996 & 119986 & 119992 & 233072 & 17142 & 235844 & 14962 & 860994 \\
\hline 11 & 138534 & 119992 & 119986 & 119988 & 239306 & 17146 & 235582 & 14960 & 866960 \\
\hline 12 & 149337 & 119994 & 119990 & 119988 & 236482 & 17144 & 236078 & 14992 & 864668 \\
\hline 13 & 149539 & 119994 & 119994 & 119994 & 246826 & 17142 & 235884 & 15004 & 874838 \\
\hline 14 & 151627 & 119984 & 119988 & 119992 & 252570 & 17142 & 235250 & 14952 & 879878 \\
\hline 15 & 160123 & 119900 & 119988 & 119994 & 241474 & 17142 & 235640 & 15008 & 869146 \\
\hline 16 & 198451 & 119988 & 119988 & 119992 & 236556 & 17142 & 235992 & 14984 & 864642 \\
\hline Avg & & 119983 & 119989 & 119992 & 238808 & 17144 & 235604 & 14822 & 866342 \\
\hline
\end{tabular}

Table 2. Mesh quality factor, $Q$, for each head compartment of every dataset.

\begin{tabular}{|c|c|c|c|c|c|c|c|c|c|}
\hline$\#$ & Head ID & $\begin{array}{c}Q_{,} \\
\text {Skin }\end{array}$ & $\begin{array}{c}Q_{,} \\
\text {skull }\end{array}$ & $\begin{array}{c}Q_{,} \\
\text {CSF }\end{array}$ & $\begin{array}{c}Q_{,} \\
\text {GM }\end{array}$ & $\begin{array}{c}Q_{,} \\
\text {cerebel. }\end{array}$ & $\begin{array}{c}Q_{,} \\
\text {WM }\end{array}$ & $\begin{array}{c}Q_{,} \\
\text {ventricles }\end{array}$ & $\begin{array}{c}Q_{,} \\
\text {avg }\end{array}$ \\
\hline 1 & 101309 & 0.18 & 0.25 & 0.21 & 0.25 & 0.19 & 0.34 & 0.51 & 0.27 \\
\hline 2 & 110411 & 0.16 & 0.17 & 0.22 & 0.20 & 0.24 & 0.30 & 0.55 & 0.26 \\
\hline 3 & 117122 & 0.15 & 0.21 & 0.18 & 0.23 & 0.35 & 0.29 & 0.37 & 0.25 \\
\hline 4 & 120111 & 0.15 & 0.18 & 0.18 & 0.17 & 0.21 & 0.23 & 0.35 & 0.21 \\
\hline 5 & 122317 & 0.15 & 0.15 & 0.20 & 0.26 & 0.24 & 0.23 & 0.46 & 0.24 \\
\hline 6 & 122620 & 0.17 & 0.25 & 0.21 & 0.25 & 0.34 & 0.22 & 0.41 & 0.27 \\
\hline 7 & 124422 & 0.20 & 0.19 & 0.22 & 0.21 & 0.36 & 0.32 & 0.54 & 0.29 \\
\hline 8 & 128632 & 0.17 & 0.22 & 0.17 & 0.22 & 0.17 & 0.17 & 0.34 & 0.21 \\
\hline 9 & 130013 & 0.18 & 0.17 & 0.14 & 0.24 & 0.39 & 0.27 & 0.30 & 0.24 \\
\hline 10 & 131722 & 0.18 & 0.21 & 0.21 & 0.18 & 0.30 & 0.20 & 0.48 & 0.25 \\
\hline 11 & 138534 & 0.15 & 0.22 & 0.19 & 0.24 & 0.32 & 0.17 & 0.38 & 0.24 \\
\hline 12 & 149337 & 0.24 & 0.16 & 0.17 & 0.22 & 0.39 & 0.33 & 0.49 & 0.28 \\
\hline 13 & 149539 & 0.24 & 0.24 & 0.24 & 0.23 & 0.16 & 0.32 & 0.52 & 0.28 \\
\hline 14 & 151627 & 0.19 & 0.16 & 0.20 & 0.19 & 0.30 & 0.31 & 0.38 & 0.25 \\
\hline 15 & 160123 & 0.17 & 0.16 & 0.16 & 0.26 & 0.31 & 0.28 & 0.34 & 0.24 \\
\hline 16 & 198451 & 0.16 & 0.18 & 0.14 & 0.25 & 0.30 & 0.25 & 0.46 & 0.25 \\
\hline Avg & & 0.18 & 0.20 & 0.19 & 0.22 & 0.29 & 0.26 & 0.43 & 0.25 \\
\hline
\end{tabular}


Table 3. Average edge length, $l, \mathrm{~mm}$ for each head compartment of every dataset.

\begin{tabular}{|c|c|c|c|c|c|c|c|c|c|}
\hline \# & Head ID & $\begin{array}{c}l, \\
\text { Skin }\end{array}$ & $\begin{array}{c}l, \\
\text { skull }\end{array}$ & $\begin{array}{c}l, \\
\mathrm{CSF}\end{array}$ & $\begin{array}{c}l, \\
\mathrm{GM}\end{array}$ & $\begin{array}{c}l, \\
\text { cerebel. }\end{array}$ & $\begin{array}{c}l, \\
\text { WM }\end{array}$ & $\begin{array}{c}l, \\
\text { ventricles }\end{array}$ & $\begin{array}{c}l, \\
\text { avg }\end{array}$ \\
\hline 1 & 101309 & 1.65 & 1.41 & 1.31 & 1.54 & 1.84 & 1.51 & 1.10 & 1.48 \\
\hline 2 & 110411 & 1.61 & 1.40 & 1.30 & 1.49 & 1.85 & 1.46 & 1.18 & 1.47 \\
\hline 3 & 117122 & 1.60 & 1.39 & 1.31 & 1.50 & 1.85 & 1.45 & 1.22 & 1.48 \\
\hline 4 & 120111 & 1.59 & 1.39 & 1.31 & 1.52 & 1.81 & 1.46 & 1.12 & 1.46 \\
\hline 5 & 122317 & 1.59 & 1.39 & 1.31 & 1.50 & 1.82 & 1.45 & 1.19 & 1.46 \\
\hline 6 & 122620 & 1.63 & 1.41 & 1.31 & 1.52 & 1.87 & 1.45 & 1.24 & 1.49 \\
\hline 7 & 124422 & 1.68 & 1.39 & 1.31 & 1.52 & 1.85 & 1.46 & 1.25 & 1.49 \\
\hline 8 & 128632 & 1.64 & 1.40 & 1.31 & 1.54 & 1.85 & 1.48 & 1.19 & 1.49 \\
\hline 9 & 130013 & 1.58 & 1.38 & 1.30 & 1.55 & 1.87 & 1.49 & 1.24 & 1.49 \\
\hline 10 & 131722 & 1.64 & 1.40 & 1.31 & 1.53 & 1.85 & 1.50 & 1.18 & 1.49 \\
\hline 11 & 138534 & 1.69 & 1.42 & 1.30 & 1.51 & 1.85 & 1.46 & 1.16 & 1.48 \\
\hline 12 & 149337 & 1.64 & 1.39 & 1.30 & 1.54 & 1.87 & 1.48 & 1.23 & 1.49 \\
\hline 13 & 149539 & 1.65 & 1.38 & 1.31 & 1.50 & 1.85 & 1.46 & 1.20 & 1.48 \\
\hline 14 & 151627 & 1.66 & 1.39 & 1.31 & 1.48 & 1.86 & 1.42 & 1.23 & 1.48 \\
\hline 15 & 160123 & 1.68 & 1.39 & 1.30 & 1.48 & 1.83 & 1.45 & 1.18 & 1.47 \\
\hline 16 & 198451 & 1.63 & 1.39 & 1.31 & 1.57 & 1.85 & 1.52 & 1.19 & 1.49 \\
\hline Avg & & 1.64 & 1.39 & 1.31 & 1.52 & 1.85 & 1.47 & 1.19 & 1.48 \\
\hline
\end{tabular}

Table 4. Average mesh resolution, $R$, points $/ \mathrm{mm}^{2}$ for each head compartment of every dataset.

\begin{tabular}{|c|c|c|c|c|c|c|c|c|c|}
\hline$\#$ & Head ID & $\begin{array}{c}R, \\
\text { skin }\end{array}$ & $\begin{array}{c}R, \\
\text { skull }\end{array}$ & $\begin{array}{c}R, \\
\text { CSF }\end{array}$ & $\begin{array}{c}R, \\
\text { GM }\end{array}$ & $\begin{array}{c}R, \\
\text { cerebel. }\end{array}$ & $\begin{array}{c}R, \\
\text { WM }\end{array}$ & $\begin{array}{c}R, \\
\text { ventricles }\end{array}$ & $\begin{array}{c}R, \\
\text { avg }\end{array}$ \\
\hline 1 & 101309 & 0.44 & 0.61 & 0.70 & 0.50 & 0.35 & 0.52 & 0.98 & 0.59 \\
\hline 2 & 110411 & 0.46 & 0.61 & 0.71 & 0.53 & 0.35 & 0.56 & 0.86 & 0.58 \\
\hline 3 & 117122 & 0.47 & 0.62 & 0.70 & 0.53 & 0.35 & 0.56 & 0.79 & 0.57 \\
\hline 4 & 120111 & 0.47 & 0.62 & 0.70 & 0.51 & 0.37 & 0.56 & 0.95 & 0.60 \\
\hline 5 & 122317 & 0.47 & 0.62 & 0.70 & 0.53 & 0.36 & 0.57 & 0.84 & 0.58 \\
\hline 6 & 122620 & 0.45 & 0.61 & 0.69 & 0.51 & 0.34 & 0.57 & 0.77 & 0.56 \\
\hline 7 & 124422 & 0.43 & 0.62 & 0.70 & 0.51 & 0.35 & 0.56 & 0.76 & 0.56 \\
\hline 8 & 128632 & 0.45 & 0.62 & 0.70 & 0.50 & 0.35 & 0.55 & 0.84 & 0.57 \\
\hline 9 & 130013 & 0.48 & 0.63 & 0.71 & 0.49 & 0.34 & 0.53 & 0.78 & 0.57 \\
\hline 10 & 131722 & 0.45 & 0.61 & 0.70 & 0.51 & 0.35 & 0.53 & 0.86 & 0.57 \\
\hline 11 & 138534 & 0.42 & 0.59 & 0.71 & 0.52 & 0.35 & 0.56 & 0.88 & 0.58 \\
\hline 12 & 149337 & 0.45 & 0.62 & 0.71 & 0.50 & 0.34 & 0.54 & 0.78 & 0.56 \\
\hline 13 & 149539 & 0.44 & 0.63 & 0.70 & 0.52 & 0.35 & 0.56 & 0.82 & 0.57 \\
\hline 14 & 151627 & 0.43 & 0.62 & 0.70 & 0.54 & 0.35 & 0.59 & 0.78 & 0.57 \\
\hline 15 & 160123 & 0.42 & 0.62 & 0.70 & 0.54 & 0.36 & 0.56 & 0.85 & 0.58 \\
\hline 16 & 198451 & 0.45 & 0.62 & 0.70 & 0.48 & 0.35 & 0.52 & 0.83 & 0.57 \\
\hline Avg & & 0.45 & 0.62 & 0.70 & 0.51 & 0.35 & 0.55 & 0.84 & 0.57 \\
\hline
\end{tabular}




\section{Conclusions}

In this paper, we have described a collection of sixteen high-resolution, 2-manifold, CAD compatible head models within the MATLAB ${ }^{\circledR}$ platform available to all interested parties for electromagnetic and acoustic simulations. Each model contains skin, skull, CSF, GM, cerebellum, WM, and ventricles head compartments and possesses an "onion" topology: the grey matter shell is a container for white matter, ventricles, and cerebellum objects, the CSF shell contains the grey matter shell, the skull shell contains the CSF shell, and finally the skin or scalp shell contains the skull shell. The models are fully compatible with ANSYS ED FEM software, CST Studio Suite, Sim4Life/SEMCAD software, and other electromagnetic software packages.

The collection is based on MRI data from the Human Connectome Project segmented using the SimNIBS 2.1/2.1.1 processing pipeline. The average number of triangular surface facets in a model is 866,000 , the average triangle quality is 0.25 , the average edge length is $1.48 \mathrm{~mm}$, and the average surface mesh density or resolution is 0.57 points per $\mathrm{mm}^{2}$.

The collection is available via MATLAB Central at https://www.mathworks.com/matlabcentral/fileexchange/69517-collection-of-sixteen-highquality-human-head-cad-models.

\section{Acknowledgements}

The authors wish to thank Dr. Guilherme Saturnino of Danish Research Centre for Magnetic Resonance (SimNIBS), Mr. Erik Lee of Northwestern University, and Dr. Jennifer Elam of Human Connectome Project, Washington University School of Medicine for help with this project. The authors are also thankful to Sim4Life staff (Zurich, Switzerland) for providing their electromagnetic simulation software. 


\section{References}

[1] Bikson M, Brunoni AR, Charvet LE, Clark VP, Cohen LG, Deng ZD, Dmochowski J, Edward DJ, Frohlich F, Kappenman ES, Lim KO, Loo C, Mantovani A, McMullen DP, Parra LC, Pearson M, Richardson JD, Rumsey JM, Sehatpour P, Sommers D, Unal G, Wassermann EM, Woods AJ, Lisanby SH. Rigor and reproducibility in research with transcranial electrical stimulation: An NIMH-sponsored workshop. Brain Stimul. 2018 May - Jun;11(3):465-480. doi: 10.1016/j.brs.2017.12.008. Epub 2017 Dec 29.

[2] Makarov SN, Noetscher GM, Yanamadala J, Piazza MW, Louie S, Prokop A, Nazarian A, and Nummenmaa A. Virtual Human Models for Electromagnetic Studies and Their Applications. IEEE Rev Biomed Eng. 2017;10:95-121. doi: 10.1109/RBME.2017.2722420.

[3] Miranda PC, Callejón-Leblic MA, Salvador R, Ruffini G. Realistic modeling of transcranial current stimulation: The electric field in the brain. Current Opinion in Biomedical Engineering. 2018;8:20-27. doi:

https://doi.org/10.1016/i.cobme.2018.09.002.

[4] SimNIBS online: http://simnibs.de/.

[5] Thielscher A, Antunes A, Saturnino GB. Field modeling for transcranial magnetic stimulation: A useful tool to understand the physiological effects of TMS?. Conf Proc IEEE Eng Med Biol Soc. 2015;222-5. doi: 10.1109/EMBC.2015.7318340.

[6] Opitz A, Paulus W, Will S, Antunes A, Thielscher A. Determinants of the electric field during transcranial direct current stimulation. Neuroimage. 2015 Apr 1;109:140-50. doi: 10.1016/j.neuroimage.2015.01.033. Epub 2015 Jan 19.

[7] Nielsen JD, Madsen KH, Puonti O, Siebner HR, Bauer C, Madsen CG, Saturnino GB, Thielscher A. Automatic skull segmentation from MR images for realistic volume conductor models of the head: Assessment of the state-of-the-art. Neuroimage. $2018 \mathrm{Jul}$ 1;174:587-598. doi: 10.1016/j.neuroimage.2018.03.001. Epub 2018 Mar 12.

[8] Htet AT, Saturnino GB, Burnham EH, Nummenmaa A, Makarov SN. Comparative Performance of the Finite Element Method and the Boundary Element Fast Multipole Method for a Canonic Problem Mimicking Transcranial Magnetic Stimulation (TMS). 2018. bioRxiv 411082; doi: https://doi.org/10.1101/411082.

[9] FreeSurfer. Online: https://surfer.nmr.mgh.harvard.edu/

[10] Fishl B. FreeSurfer. Neuroimage. 2012 Aug 15;62(2):774-81. doi: 10.1016/j.neuroimage.2012.01.021.

[11] Smith SM, Jenkinson M, Woolrich, MW, Beckmann CF, Behrens TEJ, JohansenBerg H, Bannister PR, De Luca M, Drobnjak I, Flitney DE, Niazy RK, Saunders J, Vickers J, Zhang Y, De Stefano N, Brady JM, Matthews PM. Advances in functional and structural MR image analysis and implementation as FSL. Neuroimage. 2004;23 Suppl 1:S208-19. doi: https://doi.org/10.1016/j.neuroimage.2004.07.051.

[12] Pechaud M, Jenkinson M, Smith S. BET2 - MRI-Based Estimation of Brain, Skull and Scalp Surfaces FMRIB Technical Report TR06MP1. 2006. Report XXXIII, pp. 81-87. 
[13] Iacono MI, Neufeld E, Akinnagbe E, Bower K, Wolf J, Vogiatzis Oikonomidis I, Sharma D, Lloyd B, Wilm BJ, Wyss M, Pruessmann KP, Jakab A, Makris N, Cohen ED, Kuster N, Kainz W, Angelone LM. MIDA: A Multimodal Imaging-Based Detailed Anatomical Model of the Human Head and Neck. PLoS One. 2015 Apr 22;10(4): e0124126. doi: 10.1371/journal.pone.0124126.

[14] Van Essen DC, Ugurbil K, Auerbach E, Barch D, Behrens TE, Bucholz R, Chang A, Chen L, Corbetta M, Curtiss SW, Della Penna S, Feinberg D, Glasser MF, Harel N, Heath AC, Larson-Prior L, Marcus D, Michalareas G, Moeller S, Oostenveld R, Petersen SE, Prior F, Schlaggar BL, Smith SM, Snyder AZ, Xu J, Yacoub E. The Human Connectome Project: A data acquisition perspective. NeuroImage. 2012; 62(4):2222-2231. doi: 10.1016/j.neuroimage.2012.02.018. PMID: 22366334. Online: https://db.humanconnectome.org

[15] Lee EG, Duffy W, Hadimani RL, Waris M, Siddiqui W, Islam F, et al. Investigational effect of brain-scalp distance on the efficacy of transcranial magnetic stimulation treatment in depression. IEEE Trans Magn 2016;52:52-5. https://doi.org/10.1109/TMAG.2015.2514158.

[16] Lee EG, Rastogi P, Hadimani RL, Jiles DC, Camprodon JA. Impact of non-brain anatomy and coil orientation on inter- and intra-subject variability in TMS at midline. 2018 Sep;129(9):1873-1883. doi: 10.1016/j.clinph.2018.04.749.

[17] ibid, Appendix A. Supplementary data.

[18] The Population Head Model Repository. IT'IS Foundation website. Online: https://www.itis.ethz.ch/virtual-population/regional-human-models/phm-repository/

[19] Online: http://simnibs.de/_media/docu2.1.1/documentation_2.1_command_line_tools.pdf

[20] Makarov SN, Noetscher GM, Yanamadala J, Piazza MW, Louie S, Prokop A, Nazarian A, and Nummenmaa A. Virtual Human Models for Electromagnetic Studies and Their Applications. IEEE Rev Biomed Eng. 2017;10:95-121. doi: 10.1109/RBME.2017.2722420.

[21] See pp. 24-25 of the S1200 Reference Manual. Online: https://www.humanconnectome.org/storage/app/media/documentation/s1200/HCP_S120 $\underline{0 \_ \text {Release_Reference_Manual.pdf }}$

[22] MATLAB File Exchange. Online: https://www.mathworks.com/matlabcentral/fileexchange/51200-stltools 